\title{
Handling real numbers in ILP: a step towards better behavioural clones (Extended abstract)
}

\author{
Sašo Džeroski, Ljupčo Todorovski, Tanja Urbančič \\ Institut Jožef Stefan, Jamova 39, 61111 Ljubljana, Slovenia.
}

\section{Introduction}

The automated acquisition of knowledge for dynamic systems control is receiving increasing attention among machine learning researchers $[9,1]$. Controllers can be designed by machine learning using different kinds of information in the learning process. Recent approaches make use of the existing skill of a human operator at performing a particular control task. In this case, traces of human performance serve as sources of learning examples. This approach is named behavioural cloning [7].

The task of learning a control rule for a dynamic system from examples can be formulated as follows: given examples of the form (Time, Action, State), find a functional relation between the State and Action, i.e., Action $($ Time $)=$ $f($ State(Time - Delay) ) [9]. For simplicity, we will assume no delay, i.e., Action $=f($ State $)$. The examples are derived from a successful performance trace, where the controlled system is brought from an initial to a goal state by applying a sequence of appropriate control actions.

Current experiments in behavioural cloning assume mostly the above formulation, where the goal is implicit in the performance trace. The learned controllers can thus be applied only to the very same control task that was used to generate the examples. Obviously, it is desirable to obtain controllers that are applicable to a wider range of similar tasks. A formulation of the behavioural cloning task that assumes Action $=f($ State, Goal $)$ instead of Action $=f($ State $)$ would facilitate the induction of controllers that would be more flexible in this sense.

Another important issue is the form of the function $f$. Present approaches to behavioural cloning use propositional learning and represent $f$ as a set of classification rules, a classification tree or a regression tree. Using inductive logic programming (ILP) instead of propositional learning would facilitate the use of background knowledge and would allow for a more expressive formalism for representing $f$. Given background knowledge $B$, an appropriate formulation of the behavioural cloning problem would be Action $=f_{B}($ State, Goal $)$.

The capability of handling real numbers is essential for applying ILP systems to the problem of behavioral cloning. To achieve this capability, we transform ILP problems to propositional form, using the DINUS [3] algorithm. This allows us to use propositional learning approaches that have elaborate number-handling capabilities, including systems for learning classification rules/trees and regression rules/trees. The former predict a discrete-valued class and are suitable for learning bang-bang control, while the latter predict a real-valued class and are suitable for learning continuous control. We will refer to the task of predicting a real-valued class in the presence of background knowledge as the task of relational regression. We will use DINUS with CN2 [2] to induce bang-bang control rules for an ice cube, and DINUS with RETIS [4] to solve the relational regression problem of inducing continuous control rules for the inverted pendulum. 


\section{Learning bang-bang control}

This section is concerned with the task of learning control rules for a simple dynamic system: an ice cube. The cube with mass $1 \mathrm{~kg}$ slides without friction along one dimension. The goal is to bring the cube to a pre-specified position and keep it there. The control actions, applied at regular time intervals are: push the cube right or left with force $10 \mathrm{~N}$ (bang-bang control). The particular control rule that we will try to reconstruct is shown in Figure 1. As the magnitude of the force is fixed $(10 \mathrm{~N})$, the rule only determines the direction (+ right, - left).

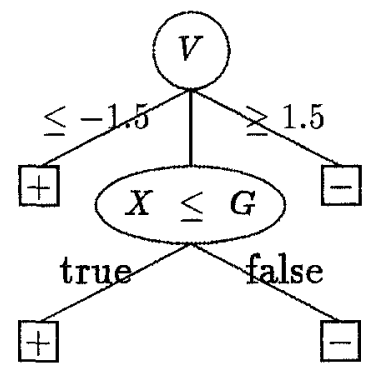

Fig. 1. A bang-bang controller for the ice cube.
If we use the formulation Action $=f($ State $)$, the task is to learn the relation cube (Action, $X, V)$, where $X[\mathrm{~m}]$ denotes the current position and $V$ $[\mathrm{m} / \mathrm{s}]$ the current velocity of the cube. To generate training examples, we used two different traces where the cube was moved from the initial states $X=-2$ and $X=5$ to the target position $G=3$. Control decisions were made every 0.02 seconds. DINUS with CN2 (as no background knowledge is present, this is equivalent to using CN2) generated the four rules below.

The rules successfully bring the cube to the particular target position from an arbitrary initial state. However, they don't work if we change the target position.

cube(left, $X, V) \leftarrow$

$X>3.00, V>-1.45$.

cube(left, $X, V) \leftarrow V>1.45$.

cube(right, $X, V) \leftarrow$

$X<3.01, V<1.45$.

cube (right, $X, V) \leftarrow V<-1.45$.
For the setting Action $=f($ State, Goal $)$, we used four behaviour traces to generate training examples: the positions $(G=-5$ and $G=3$ ) were reached from the states $(X=-2$ and $X=5$ ). The six rules below were induced. They are successful for $G=3$ and $G=-5$, but fail for other goals.

Finally, the background knowledge predicate $\operatorname{le} q(X, Y)$ (meaning $X \leq Y$ ) was used in the learning process, with the same training examples as above. DINUS transformed the ILP problem to a propositional learning problem where the class is the appropriate control action and the attributes are as follows: $X, V, G, \operatorname{leq}(X, G)$ and $\operatorname{leq}(G, X)$. The four rules below were induced.

These rules successfully bring the cube from any initial state to any target position. Having the prerequisite number handling capabilities and the appropriate background knowledge, DINUS was able to construct a successful control rule.

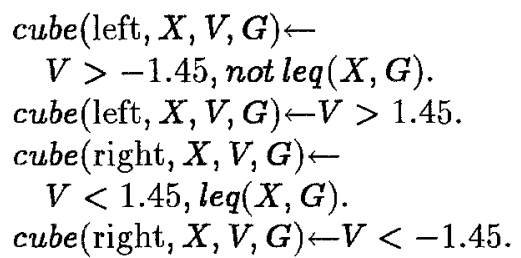




\section{Learning continuous control}

In this section, we describe how DINUS was used to induce a continuous control rule for the inverted pendulum, a standard benchmark problem for control synthesis [9]. The inverted pendulum consists of a cart that can move along a horizontal track, and a pole hinged on top of the cart, so that it can rotate in the vertical plane defined by the track and its fixed point.

The state of the cart is described with the variables $X, \dot{X}, \varphi$ and $\dot{\varphi} . X$ denotes the position of the cart, i.e., its distance from the origin point on the track, while $\varphi$ denotes the inclination angle of the pole relative to the vertical line through its fixed point. $\dot{X}$ and $\dot{\varphi}$ denote the time derivatives of $X$ and $\varphi$, i.e., the velocity of the cart and the angular velocity of the pole. A force $F$ parallel to the track can be applied to the cart. A standard control task is to bring the cart from an initial state to a particular goal position $G$ on the track, while balancing the pole at the same time.

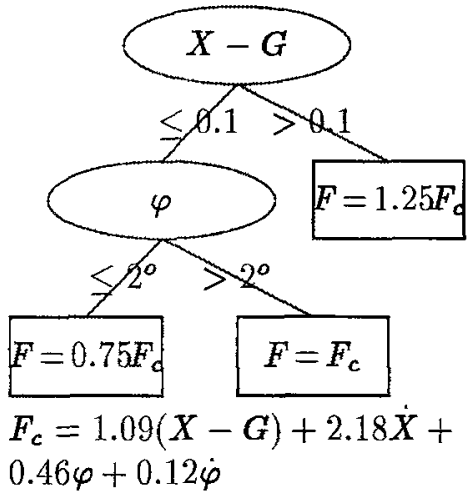

Fig. 2. A piece-wise linear controller.
The problem addressed by DINUS was to reconstruct the piece-wise linear controller shown in Figure 2. The predicate $\operatorname{diff}(X, Y, Z)$, where $Z=X-Y$, was given as background knowledge. In ILP terminology, the task was to find a definition of the target predicate $\operatorname{pole}(F, X, \dot{X}, \varphi, \dot{\varphi}, G)$. Given $X, \dot{X}, \varphi, \dot{\varphi}$, and $G$, the definition has to determine $F$. This is a relational regression problem and thus RETIS [4] was used within DINUS. RETIS induces regression trees and employs linear regression in the leaves. Using a simulator of the inverted pendulum, four behaviour traces were generated: the controller brought the cart from $X=0.5$ and $X=-0.7$ to each of $G=-0.5$ and $G=0.3$. The control force was determined by the rule in Figure 2, and was applied every 0.02 seconds. Each trace lasted five seconds, giving 250 examples per behaviour trace.

Given the target predicate and the background knowledge above, the propositional task for RETIS was to predict $F$. The attributes were $X, \dot{X}, \varphi, \dot{\varphi}, G$, and $D=X-G$. The last variable was introduced by the determinate literal $\operatorname{diff}(X, G, D)$. From the training examples generated in this manner, RETIS built a regression tree of 11 leaves, using linear regression in the leaves, without pre-pruning. Post-pruning with $m=8$ yielded the following tree:

pole $(F, X, \dot{X}, \varphi, \dot{\varphi}, G) \leftarrow \operatorname{diff}(X, G, D), D>0.1$,

$F$ is $1.4 D+2.7 X+0.58 \varphi+0.15 \dot{\varphi}$.

pole $(F, X, \dot{X}, \varphi, \dot{\varphi}, G) \leftarrow \operatorname{diff}(X, G, D), D \leq 0.1, \varphi>2.04$

$F$ is $-0.23+0.83 D+2.7 \dot{X}+0.47 \varphi+0.13 \dot{\varphi}-0.54 G$.

pole $(F, X, \dot{X}, \varphi, \dot{\varphi}, G) \leftarrow \operatorname{diff}(X, G, D), D \leq 0.1, \varphi \leq 2.04$,

$F$ is $0.82 D+1.6 \dot{X}+0.35 \varphi+0.09 \dot{\varphi}$.

The tree has the same structure as the original controller and (almost) the same numerical parameters. In addition to performing each of the four training example tasks successfully, the tree also works for other initial and goal positions. 
Further experiments have shown that learning with background knowledge produces more general, more concise and more reliable rules.

\section{Discussion}

The transformation approach, implemented in DINUS, allows for handling real numbers in ILP. In this respect, DINUS compares favorably to existing ILP systems. As compared to LINUS [6], it can use determinate new variables. Most other ILP systems do not deal with real numbers at all. Exceptions are FOIL [8] and INDLOG [1], which can use inequalities in the induced clauses. However, DINUS is the first to address the problem of relational regression and at the same time include noise handling. Both ingredients are necessary for practical applications, such as behavioural cloning. Karalič [5] is currently developing a relational regression system that builds first-order clauses to predict the value of a continuous-valued class. The system has some noise-handling capabilities and can, in principle, use linear regression.

We first generalized the formulation of the behavioral cloning task by introducing the goal state and background knowledge. Thus, behavioral cloning was formulated as an ILP task. We applied the ILP system DINUS to the problem of learning bang-bang and continuous control for two dynamic systems. The more general formulation and the use of background knowledge allowed for inducing general control rules. Extensive testing in the pole balancing domain showed that background knowledge also improves the reliability and precision of the induced rules. The induced controllers are applicable to a range of similar tasks, rather than a single specific task. Further work will address the more realistic problems of cloning human operators' skill in the domains of controlling container cranes [9] and flight simulators [1].

Acknowledgements This research is supported by the ESPRIT Project 6020 Inductive Logic Programming and the Slovenian Ministry of Science and Technology.

\section{References}

1. Camacho, R. (1994). Learning stage transition rules with Indlog. In Proc. Fourth International Workshop on Inductive Logic Programming.

2. Clark, P. and Boswell, R. (1991). Rule induction with CN2: Some recent improvements. In Proc. Fifth European Working Session on Learning.

3. Džeroski, S., Muggleton, S., and Russell, S. (1992). PAC-learnability of determinate logic programs. In Proc. Fifth ACM Workshop on Computational Learning Theory.

4. Karalič, A. (1992). Employing linear regression in regression tree leaves. In Proc. Tenth European Conference on Artificial Intelligence.

5. Karalič, A. (1994). Relational regression: first steps. Technical report IJS-DP-7001, Jožef Stefan Institute, Ljubljana.

6. Lavrač, N., Džeroski, S., and Grobelnik, M. (1991). Learning nonrecursive definitions of relations with LINUS. In Proc. Fifth European Working Session on Learning.

7. Michie, D. (1993). Knowledge, learning and machine intelligence. In L. S. Sterling (ed.) Intelligent Systems. Plenum Press, New York.

8. Quinlan, J. and Cameron-Jones, M. (1993). FOIL: A midterm report. In Proc. Sixth European Conference on Machine Learning.

9. Urbančič, T. and Bratko, I. (1994). Reconstructing human skill with machine learning. In Proc. Eleventh European Conference on Artificial Intelligence. 